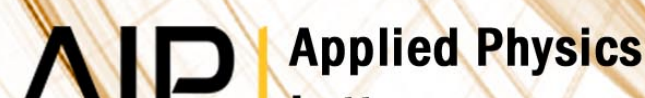 \\ Letters
}

\section{Blue light emitting diodes based on fluorescent CdSe/ZnS nanocrystals}

Aurora Rizzo, Yanqin Li, Stefan Kudera, Fabio Della Sala, Marco Zanella et al.

Citation: Appl. Phys. Lett. 90, 051106 (2007); doi: 10.1063/1.2426899

View online: http://dx.doi.org/10.1063/1.2426899

View Table of Contents: http://apl.aip.org/resource/1/APPLAB/v90/i5

Published by the American Institute of Physics.

Additional information on Appl. Phys. Lett.

Journal Homepage: http://apl.aip.org/

Journal Information: http://apl.aip.org/about/about_the_journal

Top downloads: http://apl.aip.org/features/most_downloaded

Information for Authors: http://apl.aip.org/authors

\section{ADVERTISEMENT}

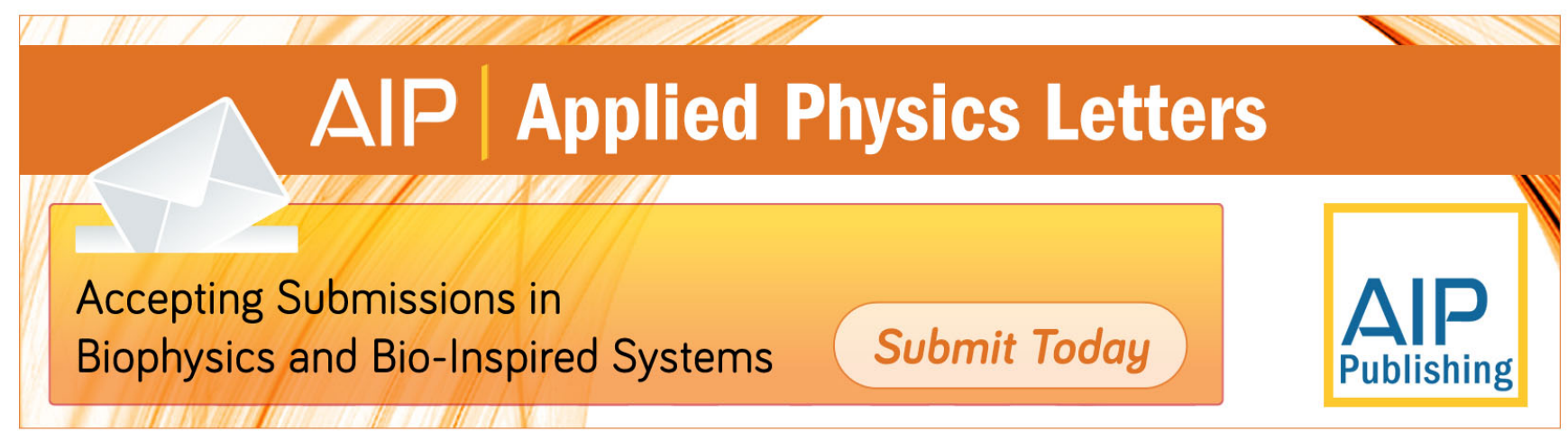




\title{
Blue light emitting diodes based on fluorescent CdSe/ZnS nanocrystals
}

\author{
Aurora Rizzo, a),b) Yanqin Li, Stefan Kudera, and Fabio Della Sala \\ National Nanotechnology Laboratory (NNL), CNR-INFM, Università degli Studi Lecce, Via per Arnesano \\ Km 5, 73100 Lecce, Italy \\ Marco Zanella and Wolfgang J. Parak \\ Center for Nanoscience, Ludwig Maximilians Universität, Amalienstraße 5480799 München, Germany \\ Roberto Cingolani, Liberato Manna, and Giuseppe Gigli ${ }^{\mathrm{c}}$ \\ National Nanotechnology Laboratory (NNL), CNR-INFM, Università degli Studi Lecce, Via per Arnesano \\ Km 5, 73100 Lecce, Italy
}

(Received 20 September 2006; accepted 28 November 2006; published online 30 January 2007)

\begin{abstract}
The authors report on the blue electroluminescence from CdSe/ZnS core/shell nanocrystals prepared from ultrasmall, magic size CdSe clusters that have a diameter of less than $2 \mathrm{~nm}$. The light emitting device consists of an active layer of nanocrystals blended with $4,4^{\prime}, N, N^{\prime}$ diphenylcarbazole and an evaporated electron transporting/hole blocking layer made of 2,9-dimethyl-4,7-diphenyl-1,10-phenanthroline. A blue, stable electroluminescence at $485 \mathrm{~nm}$ from the hybrid device was observed, in good agreement with the photoluminescence spectra of a solid film of the same nanocrystals used for the device. (c) 2007 American Institute of Physics.

[DOI: $10.1063 / 1.2426899]$
\end{abstract}

Over the last few years, colloidal semiconductor nanocrystals, also termed as colloidal quantum dots (QDs), have attracted much interest because of their high potential for optoelectronic applications such as organic light emitting diodes (OLEDs) ${ }^{1-3}$ and solar cells ${ }^{4}$. The appealing features of these materials, when properly synthesized, are their high fluorescence efficiency, ${ }^{5}$ their narrow band emission, ${ }^{5}$ their chemical stability, ${ }^{6}$ and their tunable light emission. Among the various types of fluorescent colloidal nanocrystals, core/ shell $\mathrm{CdSe} / \mathrm{ZnS}$ nanocrystals are of peculiar interest for practical applications due to the possibility to finely tune their emission wavelength in the visible spectrum by varying their size. ${ }^{5,7}$

Different approaches have been reported so far for the fabrication of light emitting diodes (LEDs) based on colloidal QDs. Hybrid organic/inorganic LEDs, for instance, were fabricated using host/guest systems consisting of a polymer doped with $\mathrm{CdSe}$ or $\mathrm{CdSe} / \mathrm{ZnS}$ nanocrystals. ${ }^{1}$ Bilayer structures were also reported which were based on a hole transporting layer made of conductive polymers and an electron transporting layer of QDs. ${ }^{2}$ Coe-Sullivan et al. have fabricated high luminance devices with electrochemical emission in the green-red region by exploiting the phase segregation between organic molecules, such as $4,4^{\prime}, N, N^{\prime}$-diphenylcarbazole (CBP) and $N, N^{\prime}$-diphenyl$N, N^{\prime}$ - bis(3-methylphenyl)-(1, 1' - biphenyl)-4,4' - diamine, and aliphatically capped QDs. ${ }^{3,7}$ In this approach the problem of poor charge conductivity of QDs, due to the insulating layer of surfactants that coat their surface, was in fact overcome elegantly by the formation of a single monolayer of QDs sandwiched between a hole and an electron transporting organic layer. This geometry separated the charge recombination process (which occurred in the QD layer) from the charge conduction process (which occurred in the two organic layers).

\footnotetext{
a) Author to whom correspondence should be addressed; FAX: 0039-0832298238.

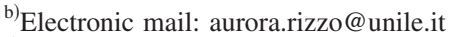

${ }^{c)}$ Electronic mail: gigli@mailing.unile.it
}

Despite the reported advances in the field, to date LEDs based on core/shell $\mathrm{CdSe} / \mathrm{ZnS}$ nanocrystals that emit pure blue light have remained difficult to fabricate. This is due to the lack of appropriate synthetic routes to synthesize CdSe nanocrystals with diameters that are sufficiently small (of the order of $1.5 \mathrm{~nm}$ ) to shift their light emission to the blue-UV region of the visible spectrum, which in addition have a narrow size distribution, good quantum efficiency, chemical stability, and which can be routinely prepared in high yields. These limitations prevent the exploitation of the nanocrystalbased technology in full-color flat display applications, for which chromophores emitting in all the three primary colors are required in order to obtain white light.

In a recent work we have reported a synthetic approach to blue emitting $\mathrm{CdSe} / \mathrm{ZnS}$ QDs which was based on the sequential growth of CdSe magic size clusters (MSCs) with progressively larger sizes. ${ }^{8}$ The method allowed us to grow extremely small colloidal CdSe nanocrystals in a mixture of three surfactants (trioctyl phosphine, dodecylamine, and nonanoic acid) at temperatures $\left(80^{\circ} \mathrm{C}\right)$ that are much lower than those involved in conventional organometallic syntheses of CdSe nanocrystals. Small nanocrystals can be grown as both nucleation and growth rates are considerably reduced, due to the large activation barriers for the two processes at such low temperature.

In order to synthesize blue emitting nanocrystals, we interrupted the growth when the largest MSCs present were the one characterized by an absorption peak at $406 \mathrm{~nm}$. These clusters, which had a diameter of approximately $1.5-1.6 \mathrm{~nm}$, were isolated from smaller MSCs present in the growth solution by size selective precipitation with methanol, ${ }^{8}$ and a $\mathrm{ZnS}$ shell was grown on them in order to passivate defects and to enhance their band-edge emission. In Fig. 1 we report the absorption and the photoluminescence spectra of core/ shell CdSe/ZnS nanocrystals that were prepared from such size-selected MSCs. In a chloroform solution the absorption and PL showed maximum peaks at 427 and $454 \mathrm{~nm}$, respectively. In the solid state, for samples prepared by drop casting the solution of nanocrystals in chloroform on a cleaned 


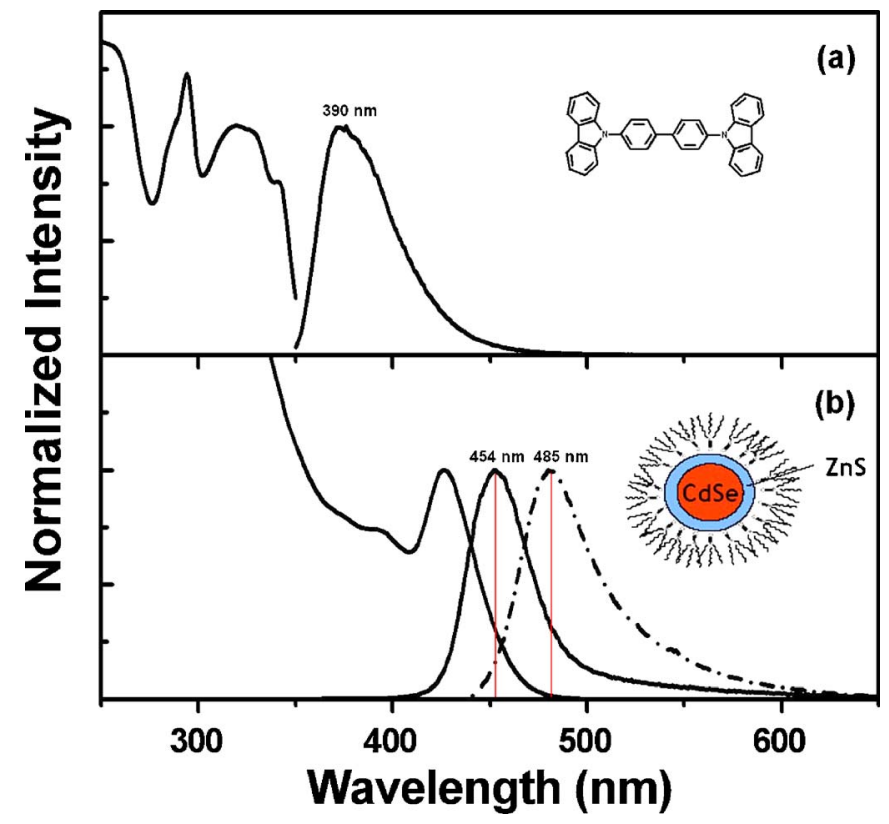

FIG. 1. (Color online) Optical properties of the materials involved in the fabrication of the LED. (a) Absorption and photoluminescence spectra of $\mathrm{CBP}$ in chloroform. (Inset) Molecular structure of CBP. (b) Absorption and photoluminescence spectra of core/shell $\mathrm{CdSe} / \mathrm{ZnS}$ nanocrystals in chloroform (solid) and in film (dash dot). (Inset) Simplified structure of a CdSe/ ZnS nanocrystal.

quartz glass, the PL spectrum showed a $30 \mathrm{~nm}$ redshift (Fig. 1). It is likely that this shift is due to the Förster energy transfer from smaller (donor) to larger (acceptor) dots within the film, and in nanocrystals it increases with increasing nonhomogeneity of the sample. ${ }^{9}$ In the present case, the process of $\mathrm{ZnS}$ shell growth leads inevitably to a broadening of the size distribution of the clusters, which is initially remarkably narrow. The Förster theory relates the efficiency of energy transfer due to donor-acceptor dipole-dipole interaction to the spectral overlap of donor emission and acceptor absorption. ${ }^{10}$ For a random orientation of transition dipoles, the rate of this process is given by

$$
k_{F}=\tau_{D}^{-1}\left(\frac{R_{0}}{r}\right)^{6}
$$

whereas the Förster radius is

$$
R_{0} \propto\left(\frac{\varphi_{D}}{n^{4}} \int_{0}^{\infty} F_{D}(\widetilde{\nu}) \varepsilon_{A}(\widetilde{\nu}) \frac{d \widetilde{\nu}}{\widetilde{\nu}^{4}}\right)^{1 / 6} .
$$

In the former expressions, $\tau_{D}$ is the lifetime of the donor in the absence of the acceptor, $r$ is the distance between the donor and the acceptor, $\varphi_{D}$ is the luminescence quantum yield of the donor, $n$ is the refractive index of the QD solid, $F_{D}(\widetilde{\nu})$ is the normalized spectrum of the donor emission, and $\varepsilon_{A}(\widetilde{\nu})$ is the molar extinction coefficient for acceptor absorption. $R_{0}$ is a measure of transfer efficiency and defines the distance at which $k_{F}$ equals the rate of donor deexcitation by competing mechanisms. In our samples, we estimated a Förster radius of about $23 \AA$ from the spectra in Fig. 1, by assuming $n \approx 2.26,{ }^{11}$ and a molar extinction coefficient at the peak of the first excited state of $2.4 \times 10^{4} \mathrm{M}^{-1} \mathrm{~cm}^{-1} .{ }^{12}$ In a recent report by Kasuya et al., the nearest-neighbor interparticle distance for close-packed films of MSCs that were very similar in size to the size-selected MSCs prepared by us was of the order of $16 \AA{ }^{13}$ Such interparticle distance certainly

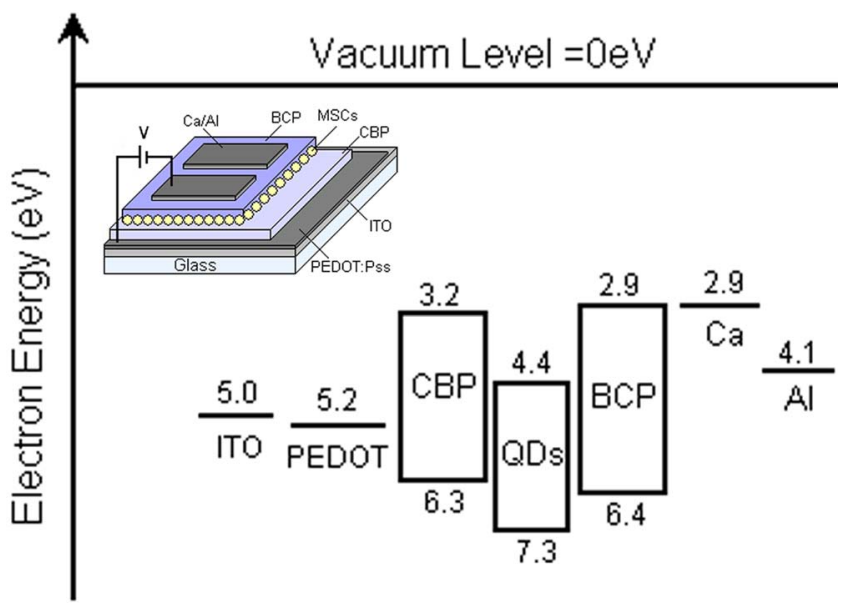

FIG. 2. (Color online) Proposed energy level diagram of the device: ITO $\|$ PEDOT: PSS $\|\mathrm{CBP}: \mathrm{MSC}\| \mathrm{BCP} \| \mathrm{Ca} / \mathrm{Al}$. (Inset) Sketch of the device.

increases in close-packed films of core-shell nanocrystals, as in the present case, but should not be larger than $20 \AA$, and is therefore shorter than the Förster radius estimated above. We want to remark, however, that the estimate of such Förster radius is certainly affected by large errors, which are due mainly to the large uncertainties in the values of the refractive index and the molar extinction coefficient for nanocrystals with such small sizes. In addition, it is likely that other effects are also responsible for the observed shift. We observed in the absorption spectrum in the film a broadening as well as a very small redshift (about $1.4 \mathrm{~nm}$ ) of the first absorption peak as compared to the spectrum recorded on the same sample in solution (data not shown). The latter effect can be justified by considering the formation of delocalized states due to the interactions among nanocrystals. ${ }^{14,15}$ The small redshift can be explained by considering the changes in the surrounding environment, ${ }^{15-17}$ such as the dielectric constant discontinuity at the QD boundary, which is known to increase the exciton ground state energy significantly. ${ }^{17}$ However, in our case the effect might be strongly reduced by the presence of the organic surfactants. ${ }^{18}$

$\mathrm{PL}$ efficiency of $\mathrm{CdSe} / \mathrm{ZnS}$ nanocrystals in chloroform solution varied from synthesis to synthesis, in a $30 \%-60 \%$ range. These blue emitting nanocrystals, dispersed in a matrix of (CBP), were used as active emissive layer in OLED devices with a multilayered structure [Fig. 2 inset]. In particular, in order to increase the hole injection from the indium tin oxide (ITO) anode into the active materials and also to improve the film forming properties, a thin layer of poly(3,4-ethylenrdioxythiophene):poly(styrenesulfonate) (PEDOT:PSS) was spin deposited onto the cleaned ITOcoated glass substrate and then heated at $110^{\circ} \mathrm{C}$ for $10 \mathrm{~min}$. The nanocrystals and the host material (CBP) were deposited in a single spin-casting step from a chloroform solution. ${ }^{19}$ To improve the electron injection in the nanocrystals layer a $35 \mathrm{~nm}$ electron transporting/hole blocking layer of 2,9dimethyl-4,7-diphenyl-1,10-phenanthroline (BCP) was evaporated. Finally, a Ca/Al $(50 \mathrm{~nm} / 150 \mathrm{~nm})$ cathode was thermal evaporated at a pressure of $4 \times 10^{-6}$ mbar using a contact shadow mask.

The electroluminescence spectrum of the device is reported in Fig. 3. It is peaked at the same wavelength as the PL spectrum, although it has a broader line shape. Differences between photo- and electroluminescence are attributed 


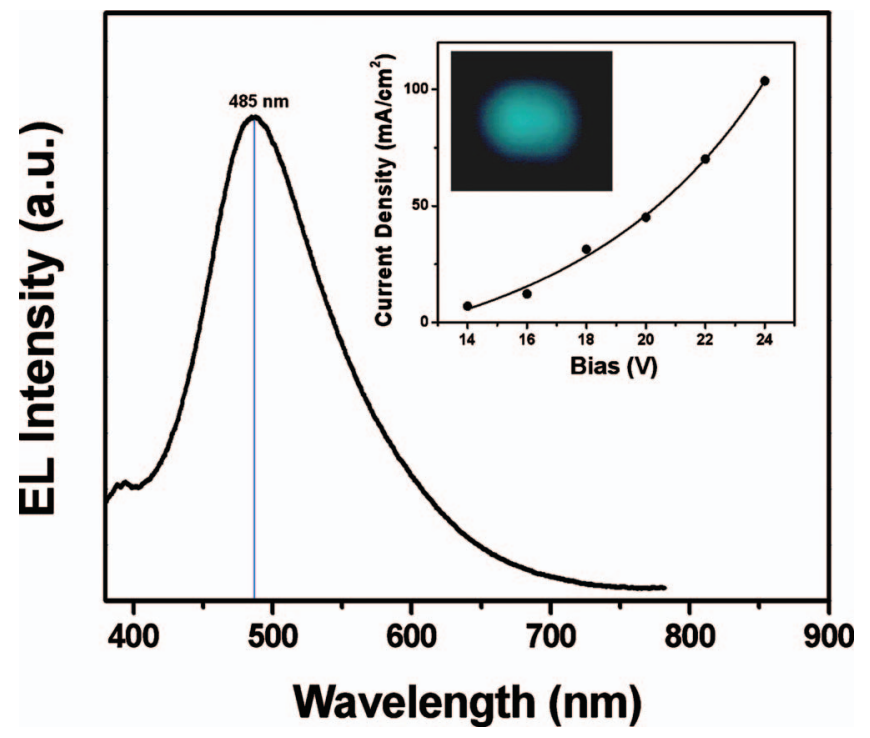

FIG. 3. (Color) Electroluminescence spectrum for the device. (Inset) $V-I$ characteristic of the device and a photo taken from the working device.

to the environmental effects, such as energy and charge transfer among nanocrystals or between nanocrystals and the organic molecules. $^{20}$ The small shoulder located at $390 \mathrm{~nm}$ from CBP is probably due to incomplete exciton energy transfer from CBP molecules to the nanocrystals. We report in Fig. 2 the energy level diagram of the device in order to clarify the processes occurring in the device. Work functions $(\Phi)$, band gaps $\left(E_{g}\right)$, ionization energies (IE), and electron affinities (EA) of ITO $(\Phi=-5.0 \mathrm{eV})$, PEDOT (IE= $-5.2 \mathrm{eV}), \mathrm{Al}(\Phi=-2.9 \mathrm{eV}), \mathrm{Ca}(\Phi=-4.1 \mathrm{eV}), \mathrm{CBP}\left(E_{g}\right.$ $=3.1 \mathrm{eV}, \quad \mathrm{IE}=-6.3 \mathrm{eV})$, and $\mathrm{BCP}\left(E_{g}=3.5 \mathrm{eV}, \mathrm{EA}=\right.$ $-2.9 \mathrm{eV})$ were taken from literature. ${ }^{1,21,22}$ The IE of the nanocrystals can be obtained approximately by adding the quantization energy to the bulk CdSe IE. ${ }^{23}$ For simplicity we treated the nanocrystals as a CdSe sphere of diameter of $1.6 \mathrm{~nm}$ and neglected the influence of the $\mathrm{ZnS}$ shell on the effective band gap. Atomistic calculations predict for this system a hole quantization energy of about $0.7 \mathrm{eV} .^{24,25}$ The resulting IE is thus $-7.3 \mathrm{eV}$, and by subtracting the nanocrystal's optical gap $(2.9 \mathrm{eV})$ estimated from the absorption measurements, we obtain a value of $-4.4 \mathrm{eV}$ for the EA.

Based on this diagram, the generation of excitons in the nanocrystals can occur either through direct charge injection or by the Förster energy transfer from the organic molecules. Electrons are injected from the $\mathrm{Ca} / \mathrm{Al}$ contact through the $\mathrm{BCP}$, due to the energy alignment of the EA of the nanocrystals and the lowest unoccupied molecular orbitals of CBP and BCP. They are eventually transported to the nanocrystals, where they are better confined due to their higher electron affinity. In charged nanocrystals the barrier for hole injection from CBP is reduced, and therefore the holes injected from the anode (ITO) through the PEDOT:PSS into the CBP layer can be trapped on the nanocrystals. By this mechanism excitons are formed on nanocrystals by direct charge injection from CBP and BCP. Excitons as well can be formed on the organic molecules placed in the interstitial spaces of the MSC layer, and then transferred to the nanocrystals by the Förster energy transfer process. According to the Förster energy transfer theory, one of the necessary conditions for an efficient process is a strong overlap of the acceptor absorp- tion and donor photoluminescence spectra. ${ }^{26,27}$ As is shown in Fig. 1 this condition is satisfied in the case of the reported device, as the PL of CBP (donor) and the absorption of nanocrystals (acceptor) are well overlapped.

In conclusion, we have fabricated a blue light emitting device based on blue emitting $\mathrm{CdSe} / \mathrm{ZnS}$ nanocrystals blended in a host material (CBP). A thin layer of a hole blocking/electron transporting material (BCP) is used to control and to balance the emission process. This work demonstrates the potentiality of colloidal semiconductor nanocrystals as blue emitters for light emitting diodes. Furthermore, when mixed with nanocrystals emitting in other colors, they can be exploited for full-color displays and lighting technology.

This work was supported by the European project SA-NANO (contract number STRP 013698) and by the Italian projects MIUR 297 (contract number 13587), FIRB Synergy (RBNE03S8XZ) and FIRB NG-LAB (contract number RBLA03ER38).

${ }^{1}$ Y. Q. Li, A. Rizzo, M. Mazzeo, L. Carbone, L. Manna, R. Cingolani, and G. Gigli, J. Appl. Phys. 97, 113501 (2005).

${ }^{2}$ H. Mattoussi, L. H. Radzilowski, B. O. Daboussi, E. L. Thomas, M. G. Bawendi, and M. F. Rubner, J. Appl. Phys. 83, 7965 (1998).

${ }^{3}$ S. Coe, W. K. Woo, M. G. Bawendi, and V. Bulovic, Nature (London) 420, 800 (2002).

${ }^{4}$ I. Gur, N. A. Fromer, M. L. Geier, and A. P. Alivisatos, Science 310, 462 (2005).

${ }^{5}$ B. O. Dabboussi, J. Rodriguez-Viejo, F. V. Mikulec, J. R. Heine, H. Mattoussi, R. Ober, K. F. Jensen, and M. G. Bawendi, J. Phys. Chem. B 101, 9463 (1997).

${ }^{6}$ V. I. Klimov, Los Alamos Sci. 28, 214 (2003).

${ }^{7}$ S. Coe-Sullivan, J. S. Steckel, W. K. Woo, M. G. Bawendi, and V. Bulovic, Adv. Funct. Mater. 15, 1117 (2005).

${ }^{8}$ S. Kudera, M. Zanella, C. Giannini, A. Rizzo, Y. Q. Li, G. Gigli, R. Cingolani, G. Ciccarella, W. Spahl, W. J. Parak, and L. Manna, Adv. Mater. (Weinheim, Ger.) (in press).

${ }^{9}$ C. R. Kagan, C. B. Murray, and M. G. Bawendi, Phys. Rev. B 54, 8633 (1996).

${ }^{10}$ T. Förster, Discuss. Faraday Soc. 27, 7 (1959).

${ }^{11}$ The refractive index for CdSe cores is calculated from the Moss rule $n^{4}=77 / E_{g}(\mathrm{eV}) ; E_{g}=2.95 \mathrm{eV}$ was estimated from the first optical absorption peak.

${ }^{12}$ W. W. Yu, L. Qu, W. Guo, and X. Peng, Chem. Mater. 15, 2854 (2003).

${ }^{13}$ A. Kasuya, R. Sivamohan, Y. A. Barnakov, I. M. Dmitruk, T. Nirasawa, V. R. Romanyuk, V. Kumar, S. V. Mamykin, K. Tohji, B. Jeyadevan, K. Shinoda, T. Kudo, O. Terasaki, Z. Liu, R. V. Belosludov, V. Sundararajan, and Y. Kawazoe, Nat. Mater. 3, 99 (2004).

${ }^{14}$ M. V. Artemyev, A. I. Bibik, L. I. Gurinovich, S. V. Gaponenko, and U. Woggon, Phys. Rev. B 60, 1504 (1999).

${ }^{15}$ H. Döllefeld, H. Weller, and A. Eychmüller, J. Phys. Chem. B 106, 5604 (2002).

${ }^{16}$ C. A. Leatherdale and M. G. Bawendi, Phys. Rev. B 63, 165315 (2001).

${ }^{17}$ V. A. Fonoberov, E. P. Pokatilov, and A. A. Balandin, Phys. Rev. B 66, 085310 (2002).

${ }^{18}$ B. S. Kim, M. A. Islam, L. E. Brus, and I. P. Herman, J. Appl. Phys. 89, 8127 (2001).

${ }^{19}$ J. S. Steckel, J. P. Zimmer, S. Coe-Sullivan, N. E. Stott, V. Bulovic, and M. G. Bawendi, Angew. Chem. 43, 2154 (2004).

${ }^{20}$ J. L. Zhao, J. Y. Zhang, C. Y. Jiang, J. Bohhenberger, T. Basche, and A. Mews, J. Appl. Phys. 96, 3206 (2004).

${ }^{21}$ I. G. Hill and A. Kahn, J. Appl. Phys. 84, 5583 (1998).

${ }^{22}$ I. G. Hill and A. Kahn, J. Appl. Phys. 86, 4515 (1999).

${ }^{23}$ A. H. Nethercot, Phys. Rev. Lett. 33, 1088 (1974).

${ }^{24}$ L. W. Wang and A. Zuger, Phys. Rev. B 53, 9579 (1996).

${ }^{25}$ S. Sapra and D. D. Sarma, Phys. Rev. B 69, 125304 (2004).

${ }^{26}$ S. E. Shaheen, B. Kippelen, and N. Peyghambarian, J. Appl. Phys. 85, 7939 (1999)

${ }^{27}$ M. Anni, L. Manna, R. Cingolani, D. Valerini, A. Cretì, and M. Lomascolo, Appl. Phys. Lett. 85, 4169 (2004). 